\title{
Characterization of the resistance to the anorectic and endocrine effects of leptin in obesity-prone and obesity-resistant rats fed a high-fat diet
}

\author{
G Tulipano $^{1 *}$, A V Vergoni ${ }^{2 *}$, D Soldi ${ }^{1}$, E E Muller ${ }^{3}$ and \\ D Cocchi ${ }^{1}$ \\ ${ }^{1}$ Department of Biomedical Sciences and Biotechnologies, University of Brescia, Brescia, Italy \\ ${ }^{2}$ Department of Biomedical Sciences, University of Modena and Reggio Emilia, Modena, Italy \\ ${ }^{3}$ Department of Pharmacology, Chemotherapy and Medical Toxicology, University of Milan, Milan, Italy \\ (Requests for offprints should be addressed to D Cocchi, Department of Biomedical Sciences and Biotechnologies, University of Brescia, Via Valsabbina 19, \\ 25123 Brescia, Italy; Email: cocchi@med.unibs.it) \\ $\left({ }^{*} \mathrm{G}\right.$ Tulipano and A V Vergoni contributed equally to this work)
}

\begin{abstract}
Leptin produced by adipocytes controls body weight by restraining food intake and enhancing energy expenditure at the hypothalamic level. The diet-induced increase in fat mass is associated with the presence of elevated circulating leptin levels, suggesting the development of resistance to its anorectic effect. Rats, like humans, show different susceptibility to diet-induced obesity. The aim of the present study was to compare the degree of leptin resistance in obesity-prone $(\mathrm{OP})$ vs obesity-resistant (OR) rats on a moderate high-fat (HF) diet and to establish if the effects of leptin on hypothalamo-pituitary endocrine functions were preserved. Starting from 6 weeks after birth, male Sprague-Dawley rats were fed on either a commercial HF diet (fat content: $20 \%$ of total calorie intake) or a standard pellet chow (CONT diet, fat content: $3 \%$ ). After 12 weeks of diet, rats fed on HF diet were significantly heavier than rats fed on CONT diet. Animals fed on HF diet were ranked according to body weight; the two tails of the distribution were called OP and OR rats respectively. A polyethylene cannula was implanted into the right ventricle of rats 1 week before central leptin administration. After 12 weeks of HF feeding, both OR and OP rats were resistant to central leptin administration $(10 \mu \mathrm{g}$, i.c.v.) (24 h calorie intake as a percent of vehicle-treated rats: CONT rats, 62 [50; 78]; OR, 93 [66; 118]; OP, 90
\end{abstract}

[70; 120] as medians and 95\% confidence intervals (CIs) of six rats for each group). Conversely, after 32 weeks of diet both OR and OP rats were partially responsive to $10 \mu \mathrm{g}$ leptin i.c.v. as compared with CONT rats $(24 \mathrm{~h}$ calorie intake as a percent of vehicle-treated rats: CONT rats, 60 [50; 67]; OR, 65 [50; 80]; OP, 80 [60; 98] as medians and $95 \%$ CIs of six rats for each group); the decrease of food intake following $200 \mu \mathrm{g}$ leptin i.p. administration was similar in all the three groups (calorie intake as a percent of vehicle-treated rats: 86 [80; 92] as median and 95\% CI). The long-term intake of HF diet caused hyperleptinemia, hyperinsulinemia and higher plasma glucose levels in OP rats as compared with CONT rats. Plasma thyroxine (T4) was lower in all the rats fed the HF diet as compared with CONT. i.c.v. administration of leptin after 32 weeks of diet restored normal insulin levels in OP rats. Moreover, leptin increased plasma T4 concentration and strongly enhanced GH mRNA expression in the pituitary of OP as well as OR rats $(180 \pm 10 \%$ vs vehicle-treated rats). In conclusion, long-term intake of HF diet induced a partial central resistance to the anorectic effect of leptin in both lean and fat animals; the neuroendocrine effects of leptin on $\mathrm{T} 4$ and $\mathrm{GH}$ were preserved.

Journal of Endocrinology (2004) 183, 289-298

\section{Introduction}

Leptin produced by adipocytes controls the daily management of body weight homeostasis by restraining food intake and enhancing energy expenditure (Kalra et al. 1999). The increase in adiposity is the outcome of a positive energy balance as a result of less energy expenditure as compared with energy intake. Remarkably, the age-related and environmentally based increase in fat mass is associated with the presence of elevated circulating leptin levels, suggesting a loss of leptin control on hypothalamic pathways regulating appetite and energy expenditure (Spiegelman \& Flier 2001). In fact, diet-induced obesity is now regarded as a status of leptin resistance (Sahu 2004). Numerous studies suggest that leptin resistance is due to leptin insufficiency in the hypothalamus as a result 
of defective transport across the blood-brain barrier (Schwartz et al. 1996, Banks et al. 1999, Burguera et al. 2000); actually, it has been clearly demonstrated that central leptin resistance may also be due to hyperleptinemia induced by exogenous leptin infusion as well as central leptin gene delivery, implying that an alteration of molecular leptin signaling pathways is involved in the development of a defective central response to the protein (Sahu 2002, Scarpace et al. 2002).

It is well known that rats, like humans, show different susceptibility to the development of diet-induced obesity, so that it is possible to select individuals with a lean phenotype despite a high calorie intake and individuals that develop obesity (Archer et al. 2003). The aim of the present study was to compare the degree of resistance to the anorectic effect of exogenous leptin in obesity-prone $(\mathrm{OP})$ vs obesity-resistant (OR) rats selected from a large number of rats fed on a moderate high-fat (HF) diet. Reportedly, leptin also mediates adaptive responses of hypothalamo-pituitary function to changes of the nutritional status (Barash et al. 1996, Chehab et al. 1996). In particular, it can be added to the list of the metabolic signals regulating growth hormone $(\mathrm{GH})$ secretion, at least in the rat. The i.c.v. administration of an antiserum to leptin abolishes GH secretion in fed rats (Carro et al. 1997); moreover, it has been shown that leptin increases GH mRNA expression acting directly on pituitary cells (Baratta et al. 2002) as well as regulating the activity of GH-releasing hormone- and somatostatin (SS)-secreting neurons in the rat hypothalamus (Cocchi et al. 1999). Leptin also activates the hypothalamo-pituitary-thyroid axis through the thyrotropin-releasing hormone (TRH)secreting neurons located in the paraventricular nucleus (PVN) (Cusin et al. 2000, Guo et al. 2004, Huo et al. 2004) and enhances the deiodinase activity converting thyroxine (T4) to triiodothyronine (T3) in peripheral tissues (Cettour-Rose et al. 2002). Both GH and thyroid hormones play a central role in the regulation of anabolic function and energy expenditure. Thus, the second aim of the study was to establish if the effects of leptin on indices of somatotroph and thyroid functions were preserved in animals made resistant to its anorectic effect, in this way supporting the recently held concept of selective leptin resistance.

\section{Materials and Methods}

\section{Animals and diet}

This study was performed according to the Italian ethics legislation governing these experiments (Ministry of Health, Decreto Legge 116/92).

Two hundred and forty male Sprague-Dawley rats (Harlan, S Pietro al Natisone, Italy) were used; they were housed in a temperature-controlled environment (21$23^{\circ} \mathrm{C}$ ) with a $12 \mathrm{~h}$ light: $12 \mathrm{~h}$ darkness cycle, with free
Table 1 Composition of the diets used in the study. The values are expressed as $\mathrm{g} / 100 \mathrm{~g}$ diet and, within brackets, as cal/100 g diet

\section{Composition}

\begin{tabular}{llll}
\hline Fat & & Carbohydrate & Protein \\
\cline { 1 - 1 } $3(28)$ & & $56(230)$ & \\
$20(184)$ & $58(238)$ & $15(78)$
\end{tabular}

Diet

Standard pellet chow

HF diet

$20(184) \quad 58(238)$

$9(99)$

access to food and water. Starting from 6 weeks after birth, two-thirds of the animals were fed on a commercial HF diet (Rieper, Bolzano, Italy), while one-third of the animals were allowed free access to standard pellet chow and served as controls (CONT). The compositions of the diets used in the study are reported in Table 1; the HF diet was characterized by a relatively low fat content compared with other experimental diets, whose fat content reaches up to $40-60 \%$ of total calorie intake (Lin et al. 2000, Ziotopoulou et al. 2000). All animals were weighed weekly throughout the experimental period. After 10 weeks, the mean body weight of rats fed on HF diet was significantly higher as compared with CONT rats. HF rats were ranked according to body weight. Animals representing the two tails of the distribution were called OP rats and OR rats respectively. Rats were also measured (nose-anal length) to obtain the obesity index (OI) of Lee

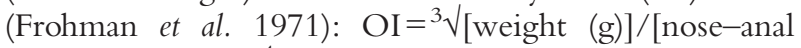
length $(\mathrm{mm})] \times 10^{4}$.

The experimental protocol is scheduled in Fig. 1. OP and $\mathrm{OR}$ rats were randomly divided for the distinct in vivo studies. For each study, an appropriate number of pairaged CONT rats were sampled randomly from the group of rats fed standard pellet chow.

\section{In vivo experiments}

A polyethylene cannula (PE-10) was implanted into the right lateral ventricle of rats, under ketamine-xylazine anesthesia, as previously described (Cocchi et al. 1999). The implantation was carried out 1 week before central administration of leptin and the correct placement of the cannula was carefully verified at the time of killing by injecting $2 \mu \mathrm{l}$ toluidine blue dye followed by decapitation and dissection of the brain. Data obtained from improperly implanted animals were discarded. Animals were housed separately. They were given $10 \mu \mathrm{g}$ recombinant mouse leptin (Sigma) in $10 \mu$ physiological saline through the cannula, just before the beginning of the dark phase of the daily darkness:light cycle. CONT rats received isovolumetric amounts of leptin vehicle. After the administration, animals were presented with a pre-weighed amount of their usual diet; 2, 4 and $24 \mathrm{~h}$ later, the residual pellets were weighed in order to calculate food consumption. The following day, rats received a second administration of 


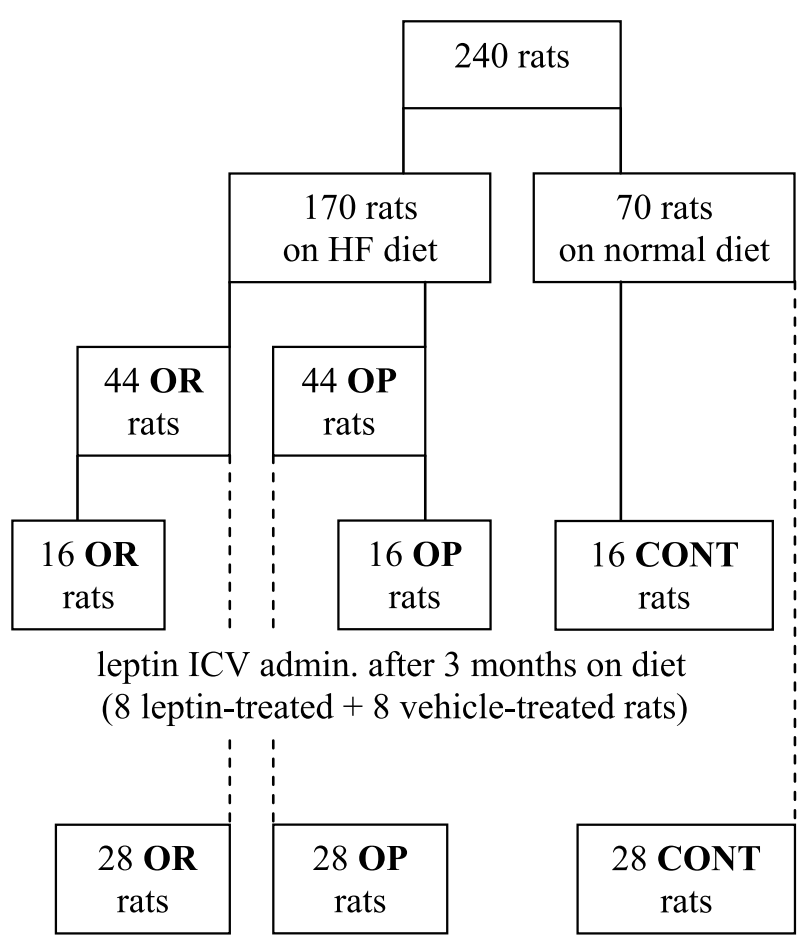

Within each group: 12 rats, leptin ip adm. (6 leptin-treated +6 vehicle treated rats);

\section{6 rats, leptin i.c.v. adm. after 6 months on diet} (8 leptin-treated +8 vehicle-treated rats)

Figure 1 Schedule of the experimental protocol.

leptin; $2 \mathrm{~h}$ later, fed rats were killed by decapitation and trunk blood was collected in EDTA-coated tubes for hormone determinations. The whole pituitary was rapidly removed from the sella turcica and the anterior lobe was rapidly dissected and frozen at $-80{ }^{\circ} \mathrm{C}$. The epididymal fat pads were removed and weighed. The study of the effects of i.p. administration of leptin $(200 \mu \mathrm{g}$ dissolved in $1 \mathrm{ml}$ physiological saline) was performed following the same time schedule of i.c.v. administration.

\section{Pituitary GH mRNA}

Total RNA was isolated from each pituitary by the singlestep acid guanidinium thiocyanate-phenol-chloroform extraction method (Trireagent; Sigma). Total RNA sample $(15 \mu \mathrm{g} / \mathrm{sample})$ from the pituitary was run on a $1 \cdot 2 \%$ formaldehyde/agarose gel and transferred to nylon membranes (Hybond N; Amersham International). Filters were hybridized with a rat GH cDNA sequence kindly provided by Dr F De Noto (University of California, San Francisco, CA, USA). The probe was labeled using the Megaprime DNA labeling system (Amersham) with $\left[\alpha_{-}{ }^{32} \mathrm{P}\right] \mathrm{dCTP}$ to a specific activity of $1 \times 10^{9}$ d.p.m. $/ \mu \mathrm{g}$ DNA. The size of the GH mRNA transcript detected by Northern hybridization, $0.8 \mathrm{~kb}$, corresponds to that reported previously (Cocchi et al. 1999). Control of the amount of the RNA loaded was performed by reprobing the filters with $\left[\alpha_{-}{ }^{32} \mathrm{P}\right] \mathrm{dCTP}-\mathrm{labeled}$ GAPDH cDNA. After hybridization, autoradiography was carried out at $-70^{\circ} \mathrm{C}$ for $12 \mathrm{~h}$ with intensifying screens using Hyperfilm-MP (Amersham).

\section{Assays for plasma insulin, T4, T3, glucose and cholesterol}

Insulin, total $\mathrm{T} 4$ and total $\mathrm{T} 3$ were assayed by RIA methods using commercial kits provided by ICN (Costa Mesa, CA, USA). Glucose was assayed by Glucotrend (Roche Diagnostics, Mannheim, Germany) and cholesterol by a colorimetric method using a commercial kit (Boehringer, Ingelheim, Germany).

\section{Statistics}

The statistical significance $(P<0 \cdot 05)$ of the differences in the mean values between the distinct experimental groups was evaluated by either a parametric or a non-parametric test when appropriate. The statistics used are described for the results in the legends of Figures and in the running text. With regard to the food intake experiments, the statistical significance refers to the difference in calorie intake between the leptin-treated rats and the respective vehicle-treated controls. Calorie intake of each leptintreated group was then depicted as percent of the calories ingested by the respective CONT group.

\section{Results}

Effects of HF diet on body weight gain

Rats fed on the HF diet gained body weight more rapidly than rats fed on standard chow. After 10 weeks of the HF diet, the mean body weight and OI of OP rats differed significantly from the values of both OR rats and CONT $\left(\mathrm{OP}\right.$ rats, $\mathrm{OI}=327 \pm 5 \cdot 7^{\mathrm{a}}$; $\mathrm{OR}$ rats, $\mathrm{OI}=308 \pm 2 \cdot 6$; CONT, OI $=300 \pm 6 \cdot 5 ;{ }^{a} P<0 \cdot 05$ vs CONT as well as OR rats, based on one-way ANOVA followed by Tukey's test). The difference in body weight between CONT and $O R$ rats reached the statistical significance after 30 weeks of the HF diet (Fig. 2A). After 32 weeks, the epididymal fat pads of $\mathrm{OP}$ rats were heavier as compared with those of CONT rats and OR rats, whereas no difference was observed between CONT and OR rats (Fig. 2B).

Rats fed on HF diet had similar calorie intake per day as compared with rats fed on standard pellet chow. A significant difference in calorie intake was observed 

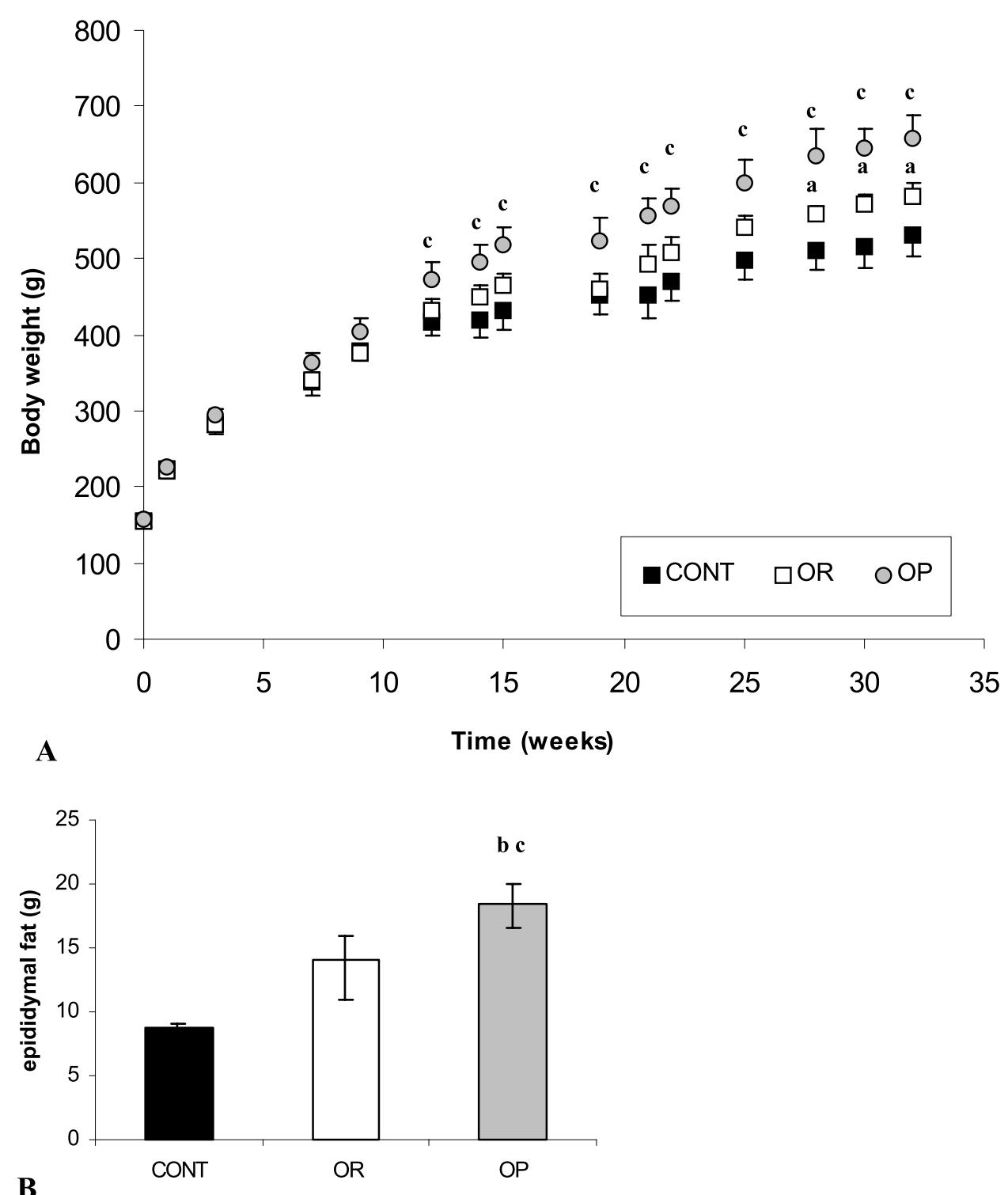

Figure 2 Rats fed on HF diet were subdivided into OR and OP rats and compared with CONT rats fed on standard chow. (A) Pattern of body weight gain during moderate HF diet feeding. Means \pm S.D. of 28 rats for each group are shown. ${ }^{a} P<0.05$ vs CONT; ${ }^{\mathrm{c}} P<0 \cdot 01$ vs CONT as well as OR (one-way ANOVA followed by Tukey's test). (B) Weight of epididymal fat pads measured after 32 weeks of HF diet. Medians and $95 \% \mathrm{Cls}$ of 12 rats for each group are shown. ${ }^{\mathrm{b}} P<0.05$ vs OR rats; ${ }^{\mathrm{c}} \mathrm{P}<0.01$ vs CONT (Kruskal-Wallis followed by Dunns' test for multiple comparisons).

between OP rats and CONT rats after 20 and 32 weeks of diet. No difference was observed between OP rats and $\mathrm{OR}$ rats at any time (Fig. 3).

Beginning from 10 weeks up to 32 weeks of diet, the increase of body weight vs time was linear $(R=0 \cdot 9)$ for each group of rats (slopes of the growth curves: CONT, $5 \cdot 802 \pm 0 \cdot 4 ; \quad$ OR, $7 \cdot 505 \pm 0 \cdot 7^{\mathrm{a}} ; \quad$ OP, $\quad 9 \cdot 548 \pm 0 \cdot 8^{\mathrm{a}, \mathrm{b}}$ ${ }^{\mathrm{a}} \mathrm{P}<0.05$ vs $\mathrm{CONT} ;{ }^{\mathrm{b}} \mathrm{P}<0 \cdot 05$ vs $\left.\mathrm{OR}\right)$. The mean calorie intake of OP rats over the same interval was $20 \%$ higher compared with CONT rats $(P<0 \cdot 05)$, whereas no difference was present between CONT and OR rats.

\section{Effects of leptin administration on energy intake}

After 12 weeks of diet, i.c.v. administration of leptin $(10 \mu \mathrm{g})$ caused a decrease of calorie intake in CONT rats; on the contrary, rats fed on HF diet were refractory to the anorectic effect of leptin (Fig. 4A). 


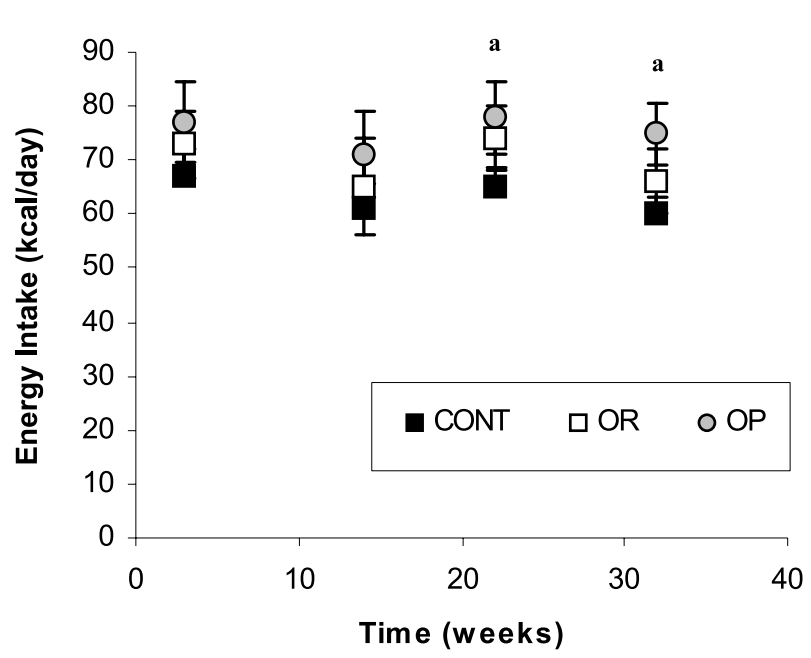

Figure 3 Energy intake measured at different growth stages. Medians and $95 \% \mathrm{Cls}$ of 12 rats for each group are shown. Animals were housed separately. ${ }^{a} P<0.05$ vs CONT

(Kruskal-Wallis followed by Dunns' test for multiple comparisons).

After 32 weeks of diet, a significant decrease of $24 \mathrm{~h}$ calorie intake was present in each experimental group following $200 \mu \mathrm{g}$ leptin given i.p. (calorie intake as percent of vehicle-treated rats: CONT, 88 [79; 94]*; OR, 82 [76; 87]*; OP, 90 [83; 95 $]^{*}$ as medians and $95 \%$ confidence intervals (CIs) of six rats; ${ }^{*} P<0 \cdot 05$ as to the decrease of calorie intake of leptin-treated rats vs the respective vehicle-treated CONT rats, based on Mann-Whitney's test). i.c.v. administration of $10 \mu \mathrm{g}$ leptin still caused a robust decrease of calorie intake in CONT rats. OR rats fed on the HF diet were still responsive to leptin but the anorectic effect of leptin was delayed and attenuated as compared with that of CONT rats; as to OP rats, the decrease of mean calorie intake did not reach statistical significance (Fig. 4B).

\section{Hormones, glucose and cholesterol determinations}

After 12 weeks of diet, plasma glucose and insulin levels were not significantly altered in rats fed on HF diet vs CONT rats. Plasma leptin tended to increase in OP animals, although not significantly (Table 2 ).

After 32 weeks of diet, plasma leptin levels measured in OP rats were significantly higher compared with that of CONT rats (CONT, 11.5 [5; 23]; OR, $20 \cdot 1$ [2; 68]; OP, $47[30-67]^{*} \mathrm{ng} / \mathrm{ml}$ as medians and $95 \%$ CIs of six rats; $* P<0.05$ vs CONT, based on Kruskal-Wallis followed by Dunns' test). The plasma concentrations of leptin were positively related to the weight of the epididymal fat pads $(R=0 \cdot 7, P<0 \cdot 0001)$. Data on insulin, thyroid hormones, glucose and cholesterol determinations are summarized in Table 3. The long-term intake of HF diet caused a significant increase of plasma insulin levels in $\mathrm{OP}$ rats compared with CONT rats whereas insulin was not altered in OR rats; plasma glucose concentrations tended to increase in OP as well as OR rats, although not significantly. HF diet decreased plasma total T4 concentrations in both OR and OP rats compared with CONT rats. i.c.v. administration of leptin significantly reduced plasma insulin concentrations in CONT rats vs the respective vehicle-treated CONT rats. Moreover, leptin administration tended to normalize plasma insulin and T4 levels in rats fed on HF diet (Dunns' test for multiple comparisons). Plasma cholesterol levels were highly variable and no changes clearly related to either HF diet intake or leptin administration were observed. The statistical analysis did not reveal any significant difference in plasma total T3 concentration related to either HF diet or treatment with leptin. The changes in metabolic and hormonal indices following systemic leptin administration to rats after 32 weeks of diet did not reach statistical significance.

Single-point determination of plasma $\mathrm{GH}$ failed to evidence any change among the different experimental groups due to the pulsatile secretion of $\mathrm{GH}$ and the ensuing high variability of GH levels (data not shown). Rats fed HF diet did not show any alterations in GH mRNA levels in the pituitary compared with CONT rats and no difference was detected between $\mathrm{OR}$ and $\mathrm{OP}$ rats. Leptin treatment of rats fed on HF diet induced a strong increase of GH mRNA expression, of the same extent in both OR and OP rats (Fig. 5).

\section{Discussion}

The association of adiposity with hypothalamic leptin resistance has been recently debated. The evidence presented in several studies suggests that in both humans and rodents the HF-feeding-induced obesity is associated with an attenuated response to the anorectic action of leptin (Sahu 2004); however, it has not been established yet how and to what extent leptin resistance may contribute to the development and the maintenance of the obese phenotype. Moreover, attempts to reverse the diet-induced obesity by leptin gene delivery into rat brain provided contradictory results, suggesting that central leptin resistance could not be considered the inevitable outcome of diet-induced obesity in rodents (Bagnasco et al. 2003, Wilsey et al. 2003).

Rats, like humans, show different susceptibility to the development of diet-induced obesity. In this context, evidence has been reported suggesting a bimodal distribution of body weight gain in rats fed an HF diet, although a recent study challenged such a conclusion, reporting a normal distribution (Archer et al. 2003). Regardless of the shape of the distribution curve, it is definitely possible to select rats having a lean phenotype despite the HF diet and rats that develop obesity (Torri et al. 2002).

The aim of the present study was to compare the degree of leptin resistance in $\mathrm{OP}$ and $\mathrm{OR}$ rats derived from a large 

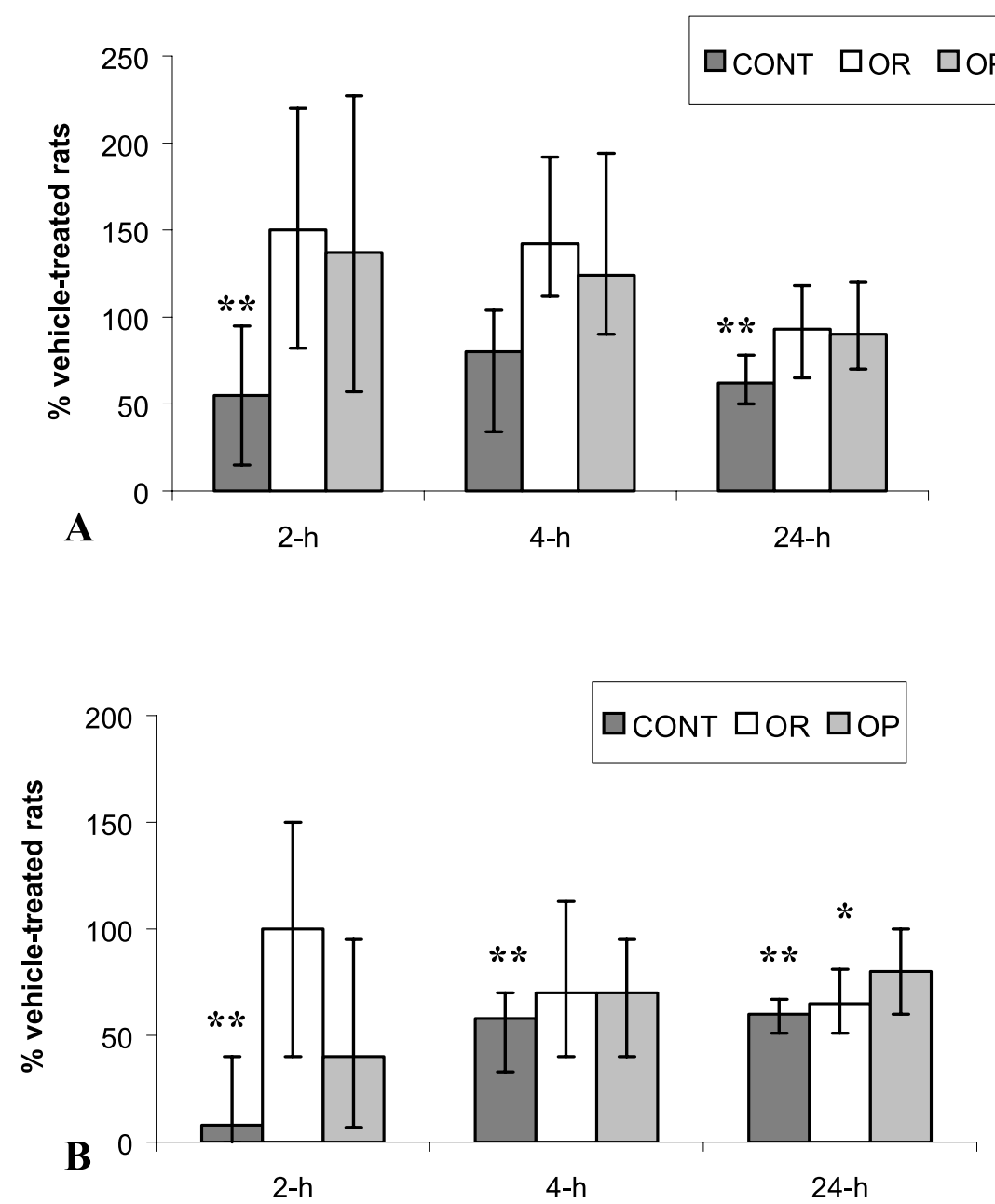

Figure 4 Effect of leptin administration on 2, 4 and $24 \mathrm{~h}$ calorie intake during the dark phase of the daily darkness:light cycle. Animals were housed separately. (A) Leptin (10 $\mu \mathrm{g})$ was administered i.c.v. to rats after a 12 week diet. (B) Leptin $(10 \mu \mathrm{g})$ was administered i.c.v. to rats after a 32 week diet. The residual food intake of leptin-treated rats is reported as a percent of calorie intake of the respective vehicle-treated CONT rats. Medians and $95 \% \mathrm{Cls}$ of six to eight rats for each group are shown. ${ }^{* *} P<0 \cdot 01$ and ${ }^{*} P<0 \cdot 05$ as to the decrease of calorie intake of leptin-treated rats vs the respective vehicle-treated CONT rats (Mann-Whitney's test).

Table 2 Plasma glucose, insulin and leptin concentrations in animals fed on either standard pellet chow (CONT) or HF diet (OR and OP) for 12 weeks. Medians and 95\% of Cls of six rats are shown

\begin{tabular}{|c|c|c|c|}
\hline & Glucose $(\mathrm{mg} / \mathrm{dl})$ & Insulin $(\mu \mathrm{IU} / \mathrm{ml})$ & Leptin $(\mathrm{ng} / \mathrm{ml})$ \\
\hline CONT rats & 116 [103; 129] & $60[52 ; 67]$ & $5.8[3.8 ; 8]$ \\
\hline OR rats & $127[123 ; 130]$ & $45[37 ; 55]$ & $7 \cdot 5[5 ; 10]$ \\
\hline OP rats & $125[105 ; 140]$ & $51[41 ; 60]$ & $10 \cdot 4[5 \cdot 4 ; 15]$ \\
\hline
\end{tabular}

cohort of rats fed chronically on a moderate HF diet, and to evaluate whether a different sensitivity to the anorectic effect of endogenous leptin may contribute to induce different phenotypes.
There was no difference in the amount of daily calorie intake ingested by the two groups of rats fed on the distinct diets. Actually, it is necessary to note that the different macroscopic composition of the two diets might have 


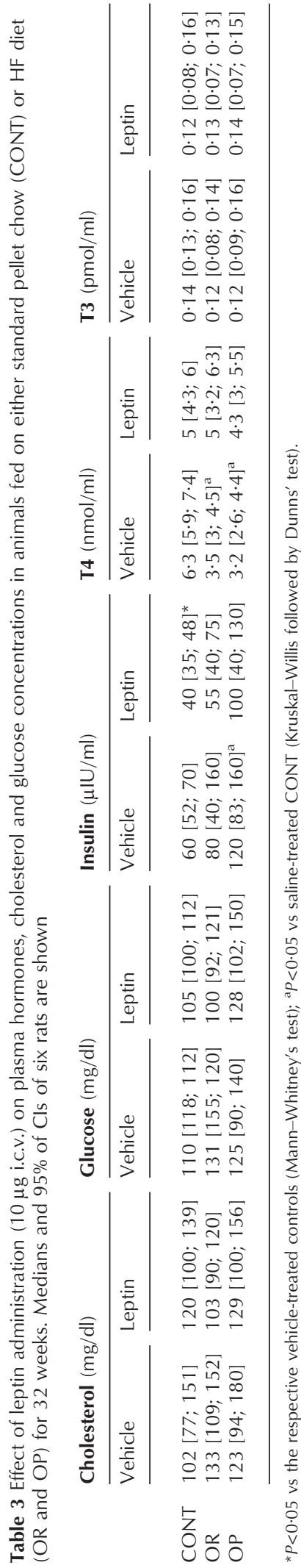


(A)

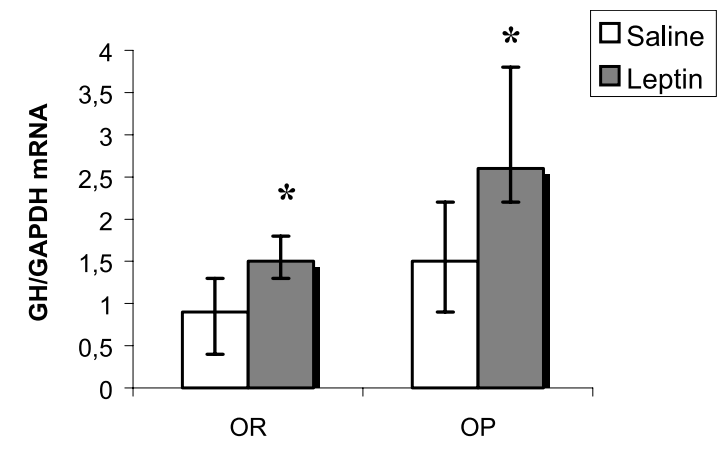

(B)

\section{GH mRNA}

\section{GAPDH mRNA}
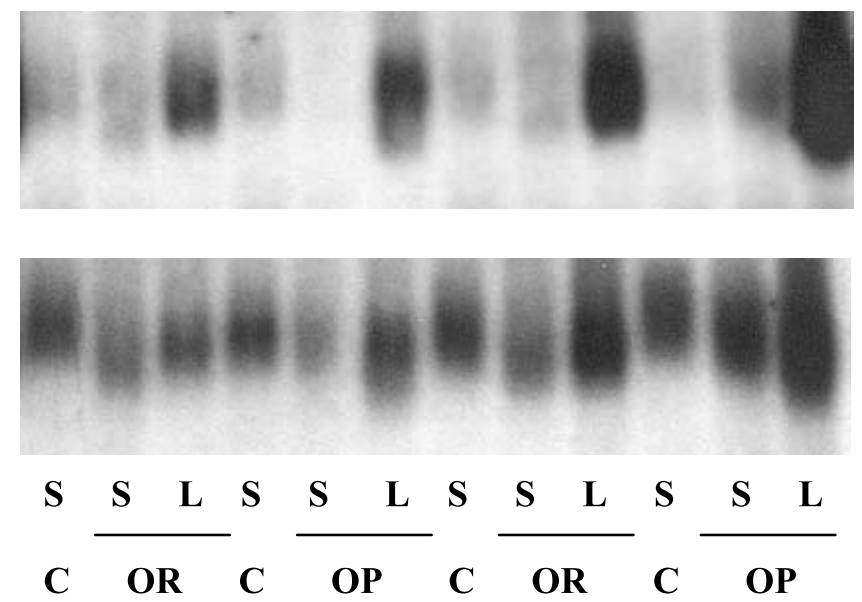

\section{Treatment}

Figure 5 Effect of leptin administration (10 $\mu$ g, i.c.v.) on pituitary GH mRNA content evaluated by Northern blot analysis in rats after 32 weeks of diet. (A) The density of the autoradiographic bands was quantified by Analytical Imaging Station FLA 2000 (Fujifilm) and the amount of GH mRNA was expressed as a percentage of GAPDH mRNA. (B) A portion of the autoradiographic sheet is shown. OR, subgroup of OR rats that were responsive to the anorectic effect of leptin; $\mathrm{OP}$, obesity prone rats; $\mathrm{C}$, rats fed standard chow; S, saline; L, leptin. Medians and $95 \% \mathrm{Cls}$ of five rats for each group. ${ }^{*} P<0.05$ vs the respective vehicle-treated CONT Rats (Mann-Whitney's test).

influenced the efficiency of nutrient uptake in the rat gastrointestinal tract. The excess of daily calorie intake of OP rats compared with that of both CONT and OR rats was rather inconsistent and by no means could account for the marked excess in body weight gain. In fact, the slope of the growth curve of OP rats from 10 to 32 weeks of diet was 1.7-fold higher compared with CONT rats whereas their mean calorie intake over the same interval was only 1.2-fold higher than that of CONT rats. Thus, likely, instrumental to the outcome of obesity was a more efficient utilization of nutrients in $\mathrm{OP}$ rats compared with OR rats associated with the intake of a high percent of fat.

After 12 weeks of diet, the rats fed on HF diet showed central resistance to the anorectic action of leptin; unexpectedly, after 32 weeks of diet central administration of leptin evidenced a restored sensitivity to the protein in OR rats, although the anorectic response to leptin was still altered in the time course and amplitude compared with that of CONT rats. Leptin tended to decrease food intake also in OP rats, although not significantly. At this stage, OP, OR and CONT animals had the same sensitivity to peripherally administered leptin.

Evidence has been presented for a defect in leptin transport across the blood-brain barrier and, consequently, for leptin access to hypothalamic nuclei, in both dietinduced obese animals as well as in obese humans (Schwartz et al. 1996, Banks et al. 1999, Burguera et al. 2000). In the present study, OP rats were as sensitive to a low dose of peripherally administered leptin as CONT rats. Actually, even in CONT rats the decrease of food intake achieved following i.p. leptin administration was 
modest. Since, reportedly, the response to peripherally administered leptin decreases with aging (Scarpace \& Turner 2001), we cannot rule out that at 32 weeks of life the brain uptake of leptin was reduced in both lean and fat rats.

To sum up, the present study implies that development of central leptin resistance associated with the continuous intake of the HF diet affects both lean and fat rats and can be divorced from the development of obesity.

Seemingly, the full desensitization of the central pathway mediating the effect of leptin on appetite may revert spontaneously to a mild form of leptin resistance without discontinuation of the HF diet. It is tempting to speculate that the high titers of plasma leptin present in rats following the prolonged HF diet intake (and aging) had induced a robust depletion of the specific blood-brain barrier transport system. Such an event, by protecting hypothalamic sites from continuous exposure to leptin would restore partial sensitivity to the protein in the central nervous system.

The partial anorectic response to leptin present in OP rats was sufficient to improve some hormonal and metabolic indices altered by the HF diet, like plasma insulin and glucose.

It is known that leptin is not only an inhibitor of food intake but also an important modulator of metabolic pathways that increase energy expenditure and reduces tissue lipids by enhancing fatty acid oxidation and inhibiting lipogenesis (Unger 2003). In this context, it is worth recalling that continuous central infusion of leptin induces an initial marked decrease in food intake, which, after 2 weeks of infusion, reverts to normality. In contrast, body weight remains stable at reduced levels despite normalization of food intake (Sahu 2002), implying that onset of tolerance to the anorectic effect of leptin is more prompt than tolerance to its metabolic effects.

In summary, revealing the peculiar features that prevent OR rats from developing an excess of body weight despite the HF diet, might suggest new solutions for the pharmacological treatment of diet-induced obesity.

Recently, to describe the preservation of the sympathoexcitatory action of leptin, despite the resistance to its satiety and weight-reducing effects, in agouti yellow obese mice and diet-induced obese mice, the concept of selective leptin resistance has been put forward (Sahu 2004). In our study, we present data further supporting the existence of selective leptin resistance in rodents fed on HF diet.

Leptin is likely to mediate adaptive responses of different neuroendocrine axes (i.e. gonadal, adrenal, thyroid and somatotrophic axis) (Barash et al. 1996, Carro et al. 1997, Guo et al. 2004) to changes of the nutritional status. In rodents, leptin stimulates GH secretion by inhibiting SS release; thus, the decrease of plasma leptin concentration may contribute to the inhibition of the GH/insulin-like growth factor (IGF)-I axis present in starved animals. The adaptive mechanisms to fasting in humans include a response of the GH/IGF-I axis which is opposite to that observed in rodents; so the possibility is raised that in humans leptin may be inhibitory and not stimulatory to $\mathrm{GH}$ secretion.

It has been previously shown that central leptin administration induces an increase of pituitary GH mRNA content in lean rats (Cocchi et al. 1999); in the present study, a clear-cut stimulatory effect of leptin on GH expression was detected in OR rats as well as in OP rats. These data suggest that a selective desensitization of the hypothalamic pathways that mediate the anorectic response to leptin in rats fed on HF diet occurs in the presence of a full sensitivity of the pathway which mediates the somatotrophic response.

A decrease of plasma T4 concentration in rats fed the HF diet was also observed in the present study, although this decrease was not accompanied by a significant change of T3 levels. It has been previously shown that leptin activates the hypothalamo-pituitary-thyroid axis through the induction of TRH gene expression in the PVN of the rat (Huo et al. 2004). In the present study, central administration of leptin reversed the decrease of $\mathrm{T} 4$ in the rats on HF diet, but it did not significantly influence T4 concentration in CONT rats. Seemingly, in CONT rats the stimulatory effect of central leptin on the thyroid axis might have been counteracted by other effects of leptin, i.e. its ability to restrict energy intake.

Reportedly, the inhibitory effect of leptin on food intake is, at least in part, mediated by the suppression of neuropeptide Y (NPY) neurons located in the arcuate nucleus and sending fibers to other distinct hypothalamic nuclei, e.g. the PVN. Thus, it has been proposed that leptin action on hypothalamo-pituitary axes might also be mediated through an effect on NPY neurons (Vaugnat et al. 1998); indeed, NPY modulates negatively the activity of TRH neurons in the PVN (Fekete et al. 2002) and is inhibitory to GH secretion in rats (Muller et al. 1999). It has been also shown that leptin can enhance directly the TRH gene expression through the activation of the JAK2-STAT3 signaling in the PVN (Huo et al. 2004) and increase directly GH mRNA content of pig somatotrophs in vitro (Baratta et al. 2002).

Overall, our data on pituitary GH expression and T4 secretion support a persistent sensitivity of pathways mediating distinct neuroendocrine effects of leptin in lean and obese rats.

\section{Funding}

This study was supported by a grant from the Italian MIUR Cofin 2000. The authors declare that there is no conflict of interest that would prejudice the impartiality of this scientific work. 


\section{References}

Archer ZA, Rayner DV, Rozman J, Klingenspor M \& Mercer JG 2003 Normal distribution of body weight gain in male SpragueDawley rats fed a high-energy diet. Obesity Research 11 1376-1383.

Bagnasco M, Dube MG, Katz A, Kalra PS \& Kalra SP 2003 Leptin expression in hypothalamic PVN reverses dietary obesity and hyperinsulinemia but stimulates ghrelin. Obesity Research 11 1463-1470.

Banks WA, DiPalma CR \& Farrel CL 1999 Impaired transport of leptin across the blood-brain barrier. Peptides $201341-1345$.

Barash IA, Cheung CC, Weigle DS, Ren H, Kabigting EB, Kujiper JL, Clifton DK \& Steiner RA 1996 Leptin is a metabolic signal to the reproductive system. Endocrinology 137 3144-3147.

Baratta M, Saleri R, Mainardi GL, Valle D, Giustina A \& Tamanini C 2002 Leptin regulates GH gene expression and secretion and nitric oxide production in pig pituitary cells. Endocrinology 143 $551-557$.

Burguera B, Couce ME, Curran GL, Jensen MD, Lloyd RV, Cleary MP \& Poduslo JF 2000 Obesity is associated with a decreased leptin transport across the blood-brain barrier in rats. Diabetes 49 1219-1223.

Carro E, Senaris R, Considine RV, Casanueva FF \& Dieguez C 1997 Regulation of in vivo growth hormone secretion by leptin. Endocrinology 138 2203-2206.

Cettour-Rose P, Burger AG, Meier CA, Visser TJ \& Rohner-Jeanrenaud F 2002 Central stimulatory effect of leptin on $\mathrm{T} 3$ production is mediated by brown adipose tissue type II deiodinase. American Journal of Physiology - Endocrinology and Metabolism 283 E980-E987.

Chehab FF, Lim ME \& Lu R 1996 Correction of the sterility defect in homozygous obese female mice by treatment with the human recombinant leptin. Nature Genetics 12 312-320.

Cocchi D, De Gennaro Colonna V, Bagnasco M, Bonacci D \& Muller EE 1999 Leptin regulates GH secretion in the rat by acting on GHRH and somatostatinergic functions. Journal of Endocrinology 162 95-99.

Cusin I, Rouro J, Visser T, Burger AG \& Rohner-Jeanrenaud F 2000 Involvement of thyroid hormones in the effect of intracerebroventricular leptin infusion on uncoupling-protein-3 expression in rat muscle. Diabetes 49 1101-1105.

Fekete C, Sarkar S, Rand WM, Harney JW, Emerson CH, Bianco AC, Beck-Sickinger A \& Lechan RM 2002 Neuropeptide Y1 and $\mathrm{Y} 5$ receptors mediate the effects of neuropeptide $\mathrm{Y}$ on the hypothalamic-pituitary-thyroid axis. Endocrinology 143 4513-4519.

Frohman LA, Goldman JK, Schnatz JD \& Bernardis LL 1971 Hypothalamic obesity in the weanling rat: effect of diet upon hormonal and metabolic alterations. Metabolism 20 501-512.

Guo F, Bakal K, Minokoshi Y \& Hollenberg AM 2004 Leptin signalling targets the thyrotropin-releasing hormone gene promoter in vivo. Endocrinology 145 2221-2227.

Huo L, Munzberg H, Nillni EA \& Bjorbaek C 2004 Role of STAT-3 in regulation of hypothalamic TRH gene-expression by leptin. Endocrinology 145 2516-2523.
Kalra SP, Dube MG, Pu S, Xu B, Horvath TL \& Kalra PS 1999 Interacting appetite-regulating pathways in the hypothalamic regulation of body weight. Endocrine Reviews 20 68-100.

Lin SH, Storlien LH \& Huang XF 2000 Leptin receptor, NPY, POMC mRNA expression in the diet-induced obese mouse brain. Brain Research 875 89-95.

Muller EE, Locatelli V \& Cocchi D 1999 Neuroendocrine control of growth hormone secretion. Physiological Reviews 79 511-607.

Sahu A 2002 Resistance to the satiety action of leptin following chronic central leptin infusion is associated with the development of leptin resistance in neuropeptide Y neurones. Journal of Neuroendocrinology 14 343-347.

Sahu A 2004 Leptin signalling in the hypothalamus: emphasis on energy homeostasis and leptin resistance. Frontiers in Neuroendocrinology 24 225-253.

Scarpace PJ \& Tumer N 2001 Peripheral and hypothalamic leptin resistance with age-related obesity. Physiology and Behavior $\mathbf{7 4}$ 721-727.

Scarpace PJ, Matheny M, Zhang Y, Tumer N, Frase CD, Shek EW, Hong B, Prima V \& Zolotukhin S 2002 Central leptin gene delivery evokes persistent leptin signal transduction in young and aged-obese rats but physiological responses become attenuated over time in aged-obese rats. Neuropharmacology 42 548-561.

Schwartz MW, Peskind E, Raskind M, Boyko EJ \& Porte D Jr 1996 Cerebrospinal fluid leptin levels: relationship to plasma levels and adiposity in humans. Nature Medicine 2 589-593.

Spiegelman BM \& Flier JS 2001 Obesity and the regulation of energy balance. Cell 104 531-543.

Torri C, Pedrazzi P, Leo G, Muller EE, Cocchi D, Agnati LF \& Zoli M 2002 Diet-induced in hypothalamic pro-opio-melanocortin mRNA in the rat hypothalamus. Peptides 23 1063-1068.

Unger RH 2003 Weapons of lean body mass destruction: the role of ectopic lipids in the metabolic syndrome. Endocrinology 144 5159-5165.

Vaugnat BAM, Pierroz DD, Lalaoui M, Englaro P, Pralong FP, Blum WF \& Aubert ML 1998 Evidence for a leptin-neuropeptide Y axis for the regulation of growth hormone secretion in the rat. Neuroendocrinology 67 291-300.

Wilsey J, Zolotukhin S, Prima V \& Scarpace PJ 2003 Central leptin gene therapy fails to overcome leptin resistance associated with diet-induced obesity. American Journal of Physiology - Regulative, Integrative and Comparative Physiology 285 R1011-R1020.

Ziotopoulou M, Mantzoros CS, Hileman SM \& Flier JS 2000 Differential expression of hypothalamic neuropeptides in the early phase of diet-induced obesity in mice. American Journal of Physiology - Endocrinology and Metabolism 279 E838-E845.

Received in final form 3 August 2004

Accepted 6 August 2004

Made available online as an Accepted Preprint

19 August 2004 